\title{
Algorithms of Channel SVD Estimation in MIMO- OFDM Systems: Evaluation and Comparison
}

\author{
M. Moghaddari \\ Communications and Computer Research Centre \\ Ferdowsi University \\ Mashhad, Iran \\ mo_mo69@stu-mail.um.ac.ir
}

\author{
H. Zamiri-Jafarian \\ Electrical Engineering Department \\ Ferdowsi University \\ Mashhad, Iran \\ hzamiri@ferdowsi.um.ac.ir
}

\begin{abstract}
The previously proposed first-order perturbationbased algorithm and linear constrained least mean square (LMS) based algorithm for channel matrix singular value decomposition (SVD) in multiple - input multiple - output (MIMO) orthogonal frequency division multiplexing (OFDM) systems are evaluated and compared in this paper from orthogonality, signal to noise plus interference ratio (SINR) and bit error rate (BER) view points. Due to approximations made in the perturbation-based algorithm to reduce its complexity, studies show that the estimates of the right and left singular matrices do not become unitary in this algorithm. This phenomenon causes more spatial interference and therefore the perturbation-based algorithm has less performance in comparison with the LMS-based algorithm at high SNRs.
\end{abstract}

Keywords- first-order perturbation-based algorithm; linear constrained LMS-based algorithm; MIMO; OFDM; SVD.

\section{INTRODUCTION}

Employing spatial diversity both in transmitter and receiver sides is a promising technique that increases bandwidth efficiency and link reliability in multiple- input multiple-output (MIMO) systems. However, intersymbol interference (ISI) and co-channel interference (CCI) are main challenging issues for exploiting the full capacity of MIMO systems.

Singular value decomposition (SVD) is a technique that is able to mitigate co-channel interference by decomposing a MIMO channel into parallel and independent single-input single-output (SISO) subchannels [1]. In addition, SVD technique provides power allocation capability that leads to full capacity utilization of the MIMO systems [2,3]. Orthogonal frequency division multiplexing (OFDM) is a pledging scheme for mitigating the ISI caused by frequency selective fading channels. Therefore, channel matrix SVD technique in company with the OFDM scheme usage would be able to overcome the previously mentioned main challenges in MIMO systems.

In this paper we evaluate and compare the performances of two SVD estimation algorithms proposed in [4] and [5] based on computational complexity, bit error rate (BER) and signal to noise plus interference (SINR) view points. In [4] an efficient adaptive algorithm is proposed in which, due to approximations made, computational complexity is reduced in comparison with the proposed algorithm in [5], however, this leads to deviation form unitary property of singular matrices. The proposed algorithm in [5] on the other hand, is based on least mean square (LMS) method with linear constraints, but it does preserve the unitary property for singular matrices.

This paper is organized as follows. After the introduction, channel model of MIMO-OFDM systems based on SVD is introduced in section II. In section III, adaptive algorithm based on first-order perturbation is described. Adaptive algorithm based on constrained LMS is reviewed in section IV. Simulation and comparison results are given in section V, followed by conclusions in section VI.

\section{Channel Model IN Mimo-OfdM Systems}

A discrete model of a MIMO-OFDM system with $N$ transmitting antennas, $M$ receiving antennas and $L$ subcarriers is shown in Fig. 1. Define $\boldsymbol{S}(k)=\left[\boldsymbol{s}^{(1)}(k), \ldots, \boldsymbol{s}^{(L)}(k)\right]$ as a transmitted OFDM symbol matrix where $\boldsymbol{s}^{(l)}(k)=\left[s_{l}^{(l)}(k), \ldots, s_{N}^{(l)}(k)\right]^{T}$ is a symbol vector transmitted from the $l$ th subcarrier. Note that $(.)^{\mathrm{T}}$ represents the transpose operation. When $\mathbf{H}=\left[\boldsymbol{H}^{(1)}, \ldots, \boldsymbol{H}^{(L)}\right]^{T}$ is an $L$-point fast Fourier transform (FFT) of the $M \times N$ channel impulse response matrix, $H(k)$, after removing the cyclic prefix, the $M \times 1$ received vector of the $l$ th subcarrier, $\boldsymbol{x}^{(l)}(k)$, becomes

$$
\boldsymbol{x}^{(l)}(k)=\boldsymbol{H}^{(l)} \boldsymbol{s}^{(l)}(k)+\boldsymbol{n}^{(l)}(k)
$$

where $\boldsymbol{H}^{(l)}$ is an $M \times N$ channel matrix of the $l$ th subcarrier and $\boldsymbol{n}^{(l)}(k)$ is an $M \times 1$ vector of the complex additive white Gaussian noise with zero-mean and autocorrelation matrix $R_{n}(k)=\sigma_{n}^{2} I_{M} \delta(k)$, while $I_{M}$ is the $M \times M$ identity matrix. The SVD of the $l$ th subcarrier channel matrix, $\boldsymbol{H}^{(l)}$, can be given as

$$
\boldsymbol{H}^{(l)}=\boldsymbol{U}^{(l)} \boldsymbol{\Sigma}^{(l)} \boldsymbol{V}^{(l) H}
$$

where $\boldsymbol{U}^{(l)}$ and $\boldsymbol{V}^{(l)}$ are $M \times P$ and $N \times P$ unitary matrices, 


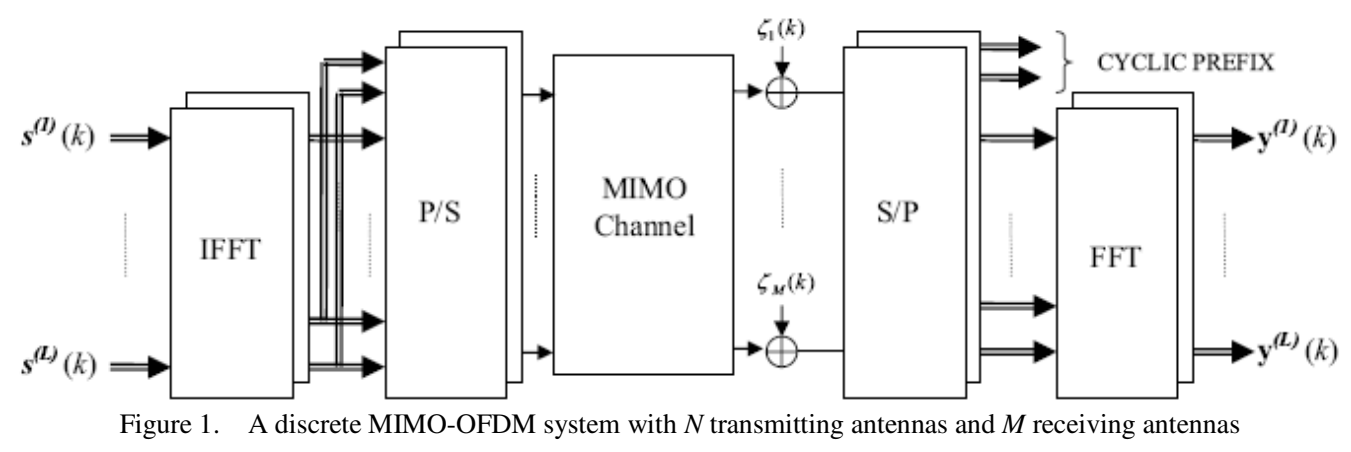

respectively. Note that $(.)^{\mathrm{H}}$ denotes transposed complex conjugate and $P$ is the rank of $\boldsymbol{H}^{(l)}$ where $P \leq \min (M, N)$. $\Sigma^{(l)}=\operatorname{diag}\left(\sigma_{1}^{(l)}, \ldots, \sigma_{P}^{(l)}\right)$ is a diagonal matrix containing the singular values of the $l$ th subcarrier channel matrix, where $\sigma_{1}^{(l)} \geq \sigma_{2}^{(l)} \geq \ldots \geq \sigma_{P}^{(l)} \geq 0$. Due to unitary property of two singular matrices, $\boldsymbol{U}^{(l)}$ and $\boldsymbol{V}^{(l)}$, estimation of the subcarrier channel SVD at the receiver side based on minimizing the mean square error criterion is a complicated procedure because of nonlinear constraints $\boldsymbol{U}^{(l)^{H}} \boldsymbol{U}^{(l)}=\boldsymbol{I}_{P}$ , $\boldsymbol{V}^{(l)^{H}} \boldsymbol{V}^{(l)}=\boldsymbol{I}_{P}$. Obtaining the singular matrices, precoding (transmitter beamforming) in transmitter side and Equalization (receiver beamforming) in receiver side can be worked out by multiplying $\boldsymbol{V}^{(l)}$ to the data vector and $\boldsymbol{U}^{(l) H}$ to the received vector, respectively. In this case, the frequency selective fading MIMO channel is converted to $L$ parallel and independent flat fading subchannels with channel gains equal to the corresponding singular values.

\section{FIRST-ORDER-PERTURBATION-BASED ALGORITHM}

Reconsidering (1) for an arbitrary subchannel at sample $k$, we have

$$
\boldsymbol{x}_{k}=\boldsymbol{H}_{k} \boldsymbol{s}_{k}+\boldsymbol{n}_{k}
$$

where $\boldsymbol{H}_{k}=\boldsymbol{U}_{k} \boldsymbol{\Sigma}_{k} \boldsymbol{V}_{k}{ }^{H}$ and $\boldsymbol{x}_{k}, \boldsymbol{s}_{k}, \boldsymbol{n}_{k}$ are $k$ th sample of received, transmitted and noise vector, respectively. Note that without loss of generality and due to simplicity, we drop the superscript of $(l)$ in the following sections. Left and right estimated singular matrices have the form $\boldsymbol{U}_{k}=\left[\boldsymbol{u}_{k, 1}, \ldots, \boldsymbol{u}_{k, P}\right]$ and $\boldsymbol{V}_{k}=\left[\boldsymbol{v}_{k, 1}, \ldots, \boldsymbol{v}_{k, P}\right]$. The true matrix $\boldsymbol{H}_{k}$ in (3) varies with time such that the change from sample $k$ to $(k+1)$ is small. Following [4], the update equation for the estimate of $\boldsymbol{H}_{k}$ can be modeled as

$$
\tilde{\boldsymbol{H}}_{k}=\alpha \tilde{\boldsymbol{H}}_{k-1}+\frac{(1-\alpha)}{\sigma_{s}^{2}} \boldsymbol{x}_{k} \boldsymbol{s}_{k}^{H}
$$

where $0 \leq \alpha \leq 1$ is the weighting factor defining the exponential decay rate of the observation window, and $k$ is the time index at which the input and output vectors are known. From (4), we can get

$$
\tilde{\boldsymbol{H}}_{k}=\tilde{\boldsymbol{H}}_{k-1}+\boldsymbol{E}
$$

where the perturbation matrix is $\boldsymbol{E}=(1-\alpha)\left(\boldsymbol{x}_{k} \boldsymbol{s}_{k}^{H}-\tilde{\boldsymbol{H}}_{k-1}\right)$. The estimated singular vectors at time index $k$ may be described in terms of small perturbations of those at $k$ - 1 , i.e., [4]

$$
\overline{\boldsymbol{U}}_{k}=\tilde{\boldsymbol{U}}_{k-1}(\boldsymbol{I}+\boldsymbol{A}), \overline{\boldsymbol{V}}_{k}=\tilde{\boldsymbol{V}}_{k-1}(\boldsymbol{I}+\boldsymbol{B})
$$

where $\boldsymbol{I}$ is the identity matrix. The overbar is used to indicate that the singular vectors are unnormalized. The diagonal elements of $\boldsymbol{A}$ and $\boldsymbol{B}$ are $a_{i i}=b_{i i}=0$, and their $(i, j)$ th elements are $a_{i j}=\tilde{\boldsymbol{u}}_{k-1, i}^{H} \tilde{\boldsymbol{u}}_{k, j}$ and $b_{i j}=\tilde{\boldsymbol{v}}_{k-1, i}^{H} \tilde{\boldsymbol{v}}_{k, j}$. It can be readily shown that, for $i<j$,

$$
\begin{aligned}
& a_{j i}=\frac{\left(\omega_{k-1, i} f_{j i}+\omega_{k-1, j} f_{i j}^{*}\right)}{\left(\omega_{k-1, i}^{2}-\omega_{k-1, j}^{2}\right)} \\
& b_{j i}=\frac{\left(\omega_{k-1, i} f_{i j}^{*}+\omega_{k-1, j} f_{j i}\right)}{\left(\omega_{k-1, i}^{2}-\omega_{k-1, j}^{2}\right)}
\end{aligned}
$$

where $f_{i j}$ is the $(i, j)$ th element of the $\boldsymbol{F}$ matrix, and $a_{j i}=-a_{i j}^{*}, b_{j i}=-b_{i j}^{*}$ for $i>j$. Also from [4]

$$
\begin{aligned}
\gamma_{k, i} & =\omega_{k-1, i}+f_{i i} \\
& =\alpha \omega_{k-1, i}+(1-\alpha) \tilde{\boldsymbol{u}}_{k-1, i}^{H} \boldsymbol{x}_{k} \boldsymbol{s}_{k}^{H} \tilde{\boldsymbol{v}}_{k-1, i}
\end{aligned}
$$

The singular value $\gamma_{k, i}$ must be real but estimation errors and noise mean that the second term in (9) may have an imaginary component, reflecting the phase offset of the $i$ th singular mode. This offset can be applied to either the left or right singular vector, hence the update is given by [4]

$$
\begin{gathered}
\omega_{k, i}=\left|\gamma_{k, i}\right| \\
\overline{\boldsymbol{u}}_{k, i} \leftarrow \gamma_{k, i} \overline{\boldsymbol{u}}_{k, i} \text { or } \overline{\boldsymbol{v}}_{k, i} \leftarrow \gamma_{k, i} \overline{\boldsymbol{v}}_{k, i}
\end{gathered}
$$

The adaptive SVD algorithm can be efficiently obtained by doing some manipulations and finally getting [4] 


$$
\begin{gathered}
\overline{\boldsymbol{u}}_{k, i}=\tilde{\boldsymbol{u}}_{k-1, i}+\frac{y_{i}^{*}}{\omega_{k-1, i}} \sum_{j=i+1}^{M} z_{j} \tilde{\boldsymbol{u}}_{n-1, j}-z_{i}^{*} \sum_{j=1}^{i-1} \frac{y_{j}}{\omega_{k-1, j}} \tilde{\boldsymbol{u}}_{k-1, j} \\
\overline{\boldsymbol{v}}_{k, i}=\tilde{\boldsymbol{v}}_{k-1, i}+\frac{z_{i}^{*}}{\omega_{k-1, i}} \sum_{j=i+1}^{N} y_{j} \tilde{\boldsymbol{v}}_{k-1, j}-y_{i}^{*} \sum_{j=1}^{i-1} \frac{z_{j}}{\omega_{k-1, j}} \tilde{\boldsymbol{v}}_{k-1, j}
\end{gathered}
$$

where $y_{i}$ and $z_{i}$ are the $i$ th elements of $\boldsymbol{y}=\sqrt{1-\alpha} \tilde{\boldsymbol{V}}_{k-1}^{H} \boldsymbol{s}_{k}$ and $\boldsymbol{z}=\sqrt{1-\alpha} \tilde{\boldsymbol{U}}_{k-1}^{H} \boldsymbol{x}_{k}$, respectively. The series of vectors $\boldsymbol{p}_{i}^{u}$ and $\boldsymbol{q}_{i+1}^{u}$ for $i=1, \ldots, M-1$, and $\boldsymbol{p}_{i}^{v}$ and $\boldsymbol{q}_{i+1}^{v}$ for $i=1, \ldots, N-1$ can be defined to make the Eq. (12) and (13) recursive, with $\boldsymbol{p}_{0}^{u}=\sqrt{1-\alpha} \boldsymbol{x}_{k}, \boldsymbol{q}_{1}^{u}=0$ and $\boldsymbol{p}_{0}^{v}=\sqrt{1-\alpha} \boldsymbol{s}_{k}, \boldsymbol{q}_{1}^{v}=0$. Finally the updated scaled singular vectors can be computed using [4]

$$
\begin{aligned}
& \overline{\boldsymbol{u}}_{k, i}=\tilde{\boldsymbol{u}}_{k-1, i}+\frac{y_{i}^{*}}{\omega_{k-1, i}} \boldsymbol{p}_{i}^{u}-z_{i}^{*} \boldsymbol{q}_{i}^{u} \\
& \overline{\boldsymbol{v}}_{k, i}=\tilde{\boldsymbol{v}}_{k-1, i}+\frac{z_{i}^{*}}{\omega_{k-1, i}} \boldsymbol{p}_{i}^{v}-y_{i}^{*} \boldsymbol{q}_{i}^{v}
\end{aligned}
$$

After updating and phase correction, the scaled vectors $\overline{\boldsymbol{u}}_{k, i}$ and $\overline{\boldsymbol{v}}_{k, i}$ should be normalized to give the singular vectors, $\boldsymbol{u}_{k, i}$ and $\boldsymbol{v}_{k, i}$.

\section{LINEAR CONSTRAINED LMS-BASED ALGORITHM}

A two-step recursive method is used in this algorithm to estimate the SVD of the channel matrix directly from the received signal. By dropping the superscript (l), (3) can be rewritten as

$$
\boldsymbol{x}(k)=\boldsymbol{H}(k) \boldsymbol{s}(k)+\boldsymbol{n}(k)
$$

we define $\boldsymbol{W}_{1}$ and $\boldsymbol{W}_{2}$ as [5]

$$
\begin{aligned}
& \boldsymbol{W}_{1}=\boldsymbol{U} \boldsymbol{\Sigma} \\
& \boldsymbol{W}_{2}=\boldsymbol{V} \boldsymbol{\Sigma}
\end{aligned}
$$

one can easily show that [5]

$$
\begin{gathered}
\boldsymbol{w}_{1 i}=\boldsymbol{H} \boldsymbol{v}_{i}=\sigma_{i} \boldsymbol{u}_{i} \\
\boldsymbol{w}_{2 i}^{H}=\boldsymbol{u}_{i}^{H} \boldsymbol{H}=\sigma_{i} \boldsymbol{v}_{i}^{H}
\end{gathered}
$$

where $\boldsymbol{w}_{1 i}, \boldsymbol{w}_{2 i}, \boldsymbol{u}_{i}$ and $\boldsymbol{v}_{i}$ are the $i$ th columns of $\boldsymbol{W}_{1}, \boldsymbol{W}_{2}$, $\boldsymbol{U}$ and $\boldsymbol{V}$, respectively. We assume that the training sequence is an independent and identically distributed (iid) signal such that $\mathrm{E}\left[s(k) s(k)^{H}\right]=\sigma_{s}^{2} I_{N}$ for all subcarriers. Note that $\mathrm{E}\left[s(k) n(j)^{H}\right]=0$ for all $k$ and $j$. By assuming $\sigma_{s}^{2}=1$, one can show that

$$
\boldsymbol{w}_{1 i}=\mathrm{E}\left[\boldsymbol{x}(k) \boldsymbol{s}(k)^{H} \boldsymbol{v}_{i}\right] \text { for } i=1, \ldots, P
$$

In the first step, by receiving $\boldsymbol{x}(k)$ and assuming $\hat{\boldsymbol{v}}_{i}(k-1)$ is the estimated $v_{i}$ at time $k-1$, the improved estimation of $\boldsymbol{w}_{1 i}$ at time $k-1, \hat{\boldsymbol{w}}_{1 i}^{+}(k-1)$, based on the estimation of $\boldsymbol{w}_{1 i}$ at time $k-1$ becomes

$$
\hat{\boldsymbol{w}}_{1 i}^{+}(k-1)=\hat{\boldsymbol{w}}_{1 i}(k-1)+\mu_{l}\left(\boldsymbol{x}(k) \mathbf{s}(k)^{H} \hat{\boldsymbol{v}}_{1 i}(k-1)-\hat{\boldsymbol{w}}_{1 i}(k-1)\right)
$$

where $\mu_{1}$ is a positive scalar step-size of the LMS algorithm. Due to the orthogonality property of the column vectors of $\boldsymbol{W}_{1}$, the estimation of $\boldsymbol{w}_{1 i}$ at time $k, \hat{\boldsymbol{w}}_{1 i}(k)$, should be obtained under the following constraint

$$
\hat{\boldsymbol{w}}_{1 j}(k)^{H} \hat{\boldsymbol{w}}_{1 i}(k)=0 \quad \forall j<i
$$

Defining [5]

$$
\begin{aligned}
& \hat{\boldsymbol{w}}_{1 i}(k)=\hat{\boldsymbol{w}}_{1 i}^{+}(k-1)+\boldsymbol{\eta}_{1 i}(k) \quad \forall i=1, \ldots, P \\
& \hat{\boldsymbol{W}}_{1 i}(k)=\left[\hat{\boldsymbol{w}}_{11}(k), \ldots, \hat{\boldsymbol{w}}_{1 i-1}(k)\right] \quad \forall i=1, \ldots, P
\end{aligned}
$$

where $\boldsymbol{\eta}_{1 i}(k)$ is a $M \times 1$ vector, $\hat{\boldsymbol{w}}_{1 i}(k)$ is obtained under the defined constraint (23) so that $\boldsymbol{\eta}_{1 i}(k)^{H} \boldsymbol{\eta}_{1 i}(k)$ is minimized [6]. From (23) and (25), we have

$$
\hat{\boldsymbol{W}}_{1 i}(k)^{H} \hat{\boldsymbol{w}}_{1 i}(k)=0 \quad \forall i=1, \ldots P
$$

Using the Lagrange multiplier method and by doing some manipulations, one can show that $\boldsymbol{w}_{1 i}$ at time $k$ can be estimated by [5]

$$
\begin{aligned}
\hat{\boldsymbol{w}}_{1 i}(k) & =\left(\boldsymbol{I}_{M}-\hat{\boldsymbol{W}}_{1 i}(k)\left(\hat{\boldsymbol{W}}_{1 i}(k)^{H} \hat{\boldsymbol{W}}_{1 i}(k)\right)^{-1} \hat{\boldsymbol{W}}_{1 i}(k)\right) \\
& \times\left(\hat{\boldsymbol{w}}_{1 i}(k-1)+\mu_{1}\left(\boldsymbol{x}(k) \boldsymbol{s}(k)^{H} \hat{\boldsymbol{v}}_{1 i}(k-1)-\hat{\boldsymbol{w}}_{1 i}(k-1)\right)\right)
\end{aligned}
$$

Based on (19), the estimation of $\boldsymbol{u}_{i}$ at time $k$ is given by

$$
\hat{\boldsymbol{u}}_{i}(k)=\left(\hat{\boldsymbol{w}}_{1 i}(k)^{H} \hat{\boldsymbol{w}}_{1 i}(k)\right)^{-\frac{1}{2}} \hat{\boldsymbol{w}}_{1 i}(k)
$$

Having $\hat{\boldsymbol{u}}_{i}(k)$ from (28), in the second step, $\boldsymbol{w}_{2 i}$ at time $k$ can be estimated with similar procedure of estimating $\boldsymbol{w}_{1 i}$ and the improved estimation of $\boldsymbol{w}_{2 i}$ at time $k-1, \hat{\boldsymbol{w}}_{2 i}^{+}(k-1)$ can be given by [5]

$$
\hat{\boldsymbol{w}}_{2 i}^{+}(k-1)=\hat{\boldsymbol{w}}_{2 i}(k-1)+\mu_{2}\left(\boldsymbol{s}(k) \boldsymbol{x}(k)^{H} \hat{\boldsymbol{u}}_{i}(k)-\hat{\boldsymbol{w}}_{2 i}(k-1)\right)
$$

where $\hat{\boldsymbol{u}}_{i}(k)$ is obtained from the first step, $\boldsymbol{x}(k)$ is the received signal at time $k$ and $\mu_{2}$ is a positive scalar step-size. Following the same procedure for $\boldsymbol{w}_{2 i}$, one can readily obtain the dual forms for Eq. (23) to (25), and finally get [5]

$$
\begin{aligned}
\hat{\boldsymbol{w}}_{2 i}(k) & =\left(\boldsymbol{I}_{N}-\hat{\boldsymbol{W}}_{2 i}(k)\left(\hat{\boldsymbol{W}}_{2 i}(k)^{H} \hat{\boldsymbol{W}}_{2 i}(k)\right)^{-1} \hat{\boldsymbol{W}}_{2 i}(k)\right) \\
& \times\left(\hat{\boldsymbol{w}}_{2 i}(k-1)+\mu_{2}\left(\boldsymbol{s}(k) \boldsymbol{x}(k)^{H} \hat{\boldsymbol{u}}_{i}(k)-\hat{\boldsymbol{w}}_{2 i}(k-1)\right)\right)
\end{aligned}
$$

By using $\hat{\boldsymbol{w}}_{2 i}(k)$ from (30), $\sigma_{i}$ and $\boldsymbol{v}_{i}$ at time $k$ are estimated by 


$$
\begin{gathered}
\hat{\sigma}_{i}(k)=\left(\hat{\boldsymbol{w}}_{2 i}(k)^{H} \hat{\boldsymbol{w}}_{2 i}(k)\right)^{\frac{1}{2}} \\
\hat{\boldsymbol{v}}_{i}(k)=\left(\hat{\boldsymbol{w}}_{2 i}(k)^{H} \hat{\boldsymbol{w}}_{2 i}(k)\right)^{-\frac{1}{2}} \hat{\boldsymbol{w}}_{2 i}(k)
\end{gathered}
$$

\section{COMPUTER Simulations}

A MIMO-OFDM system with $L=64$ for different $N$ and $M$ is considered in the simulations when the channel impulse response, $H(k)$, has an exponential delay spread profile with duration of $L_{C}=16$ that is equal to the cyclic prefix interval. The elements of the $H(k)$ are mutually independent complex Gaussian random variables with zero-mean. For each subcarrier, random symbols of BPSK, QPSK, 16QAM and 64QAM with uniform distribution are being used. In each simulation, one hundred independent channel impulse response matrices are generated for performance evaluation of each algorithm. Using the estimated precodig and equalization (right and left singular) matrices and due to unitary property of them, we have

$$
\hat{\boldsymbol{U}}^{H} \boldsymbol{H} \hat{\boldsymbol{V}}=\hat{\Sigma}
$$

where $\hat{\Sigma}$ must be a diagonal matrix with elements equal to the singular values of the channel matrix. But due to inaccuracy of estimations, off-diagonal elements of $\hat{\Sigma}$ are not exactly zero. To evaluate the diagonal property of $\hat{\Sigma}$, we define SINR as

$$
S I N R=\frac{P_{s}}{P_{I}+P_{n}}
$$

where $P_{n}$ is noise power, $P_{s}=\sum_{i=1}^{P} \hat{\sigma}_{i i}^{2}$ is signal power, and $P_{I}=\sum_{i=1}^{P} \sum_{j=1, j \neq i}^{P} \hat{\sigma}_{i j}^{2}$ represents the interference power caused by inaccuracy. As seen in Fig. 2, by increasing SNR, the interference is also increasing and make the curves saturate after $S N R=30 \quad d B$. In Fig. 3 the spatial interference in perturbation-based algorithm is considered as the ratio of sum of the energies of the main diagonal elements of $\tilde{U}^{H} \tilde{\boldsymbol{U}}$, to sum of the energies of the off-diagonal elements. In LMSbased algorithm, due to preservation of unitary property, this ratio is infinite, so it has not been depicted, however for perturbation-based algorithm the curve saturates after $S N R=$ $30 \mathrm{~dB}$. For both algorithms, the BER versus SNR for $B P S K$ and QPSK and also 16QAM and 64QAM modulation schemes are depicted in Fig. 4 and Fig. 5, respectively. As seen in these figures, the LMS-based algorithm outperforms the perturbation-based algorithm at high SNRs, specially for high constellation-point modulation schemes. However, in low SNRs two algorithms perform similar to the true case of knowing the MIMO communication channel. Also, as the number of constellation points increases, the BER of two algorithms deviate from ideal state (knowing the channel matrix) which represents the higher sensibility of 16QAM and 64QAM modulation schemes to the estimation errors.

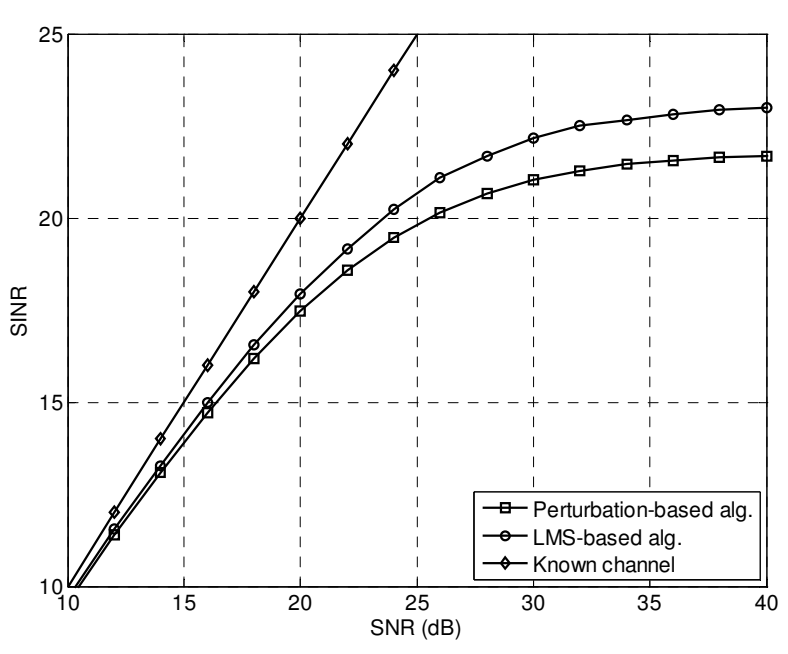

Figure 2. SINR versus SNR for $M=N=4$.

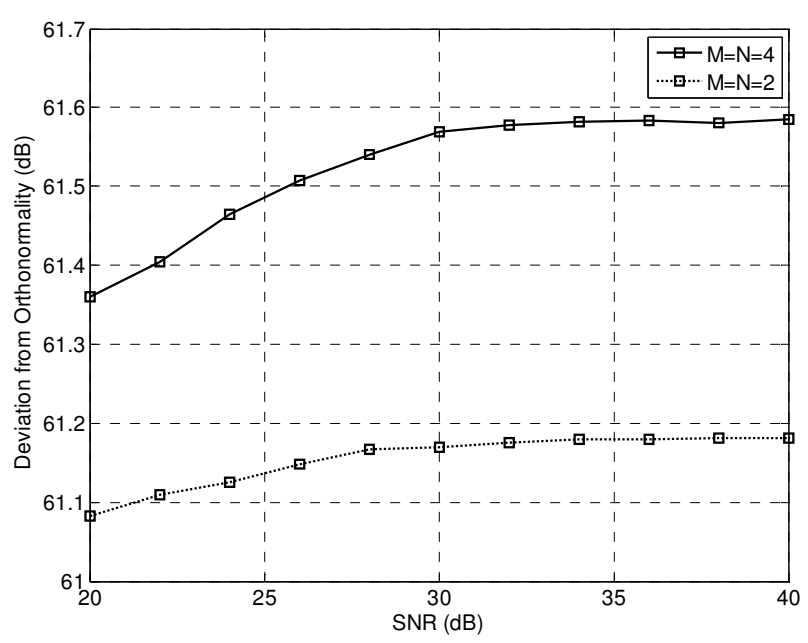

Figure 3. Deviation from orthonormality for $M=N=2$ and $M=N=4$ for perturbation-based algorithm.

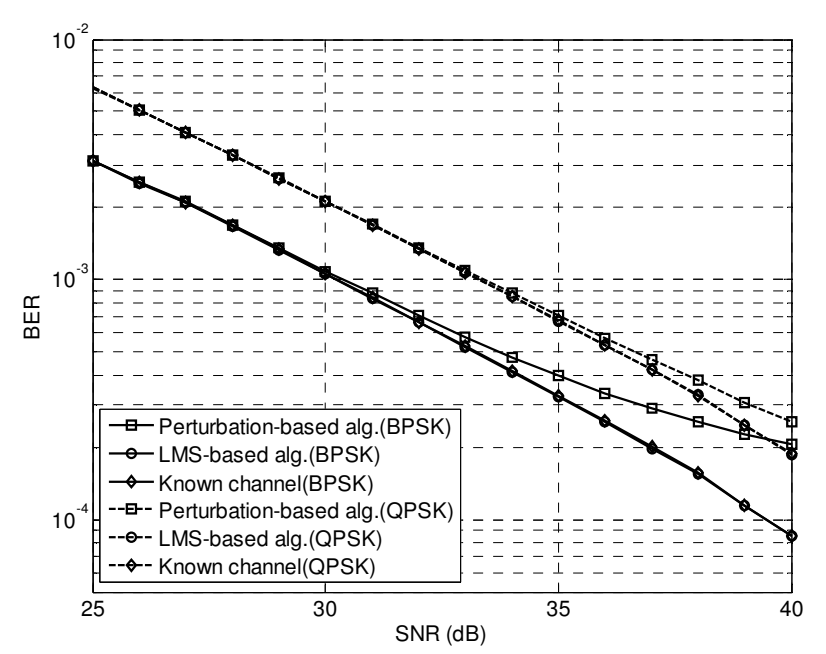

Figure 4. BER versus SNR for $B P S K$ and $Q P S K$ modulations with $M=N=4$. 


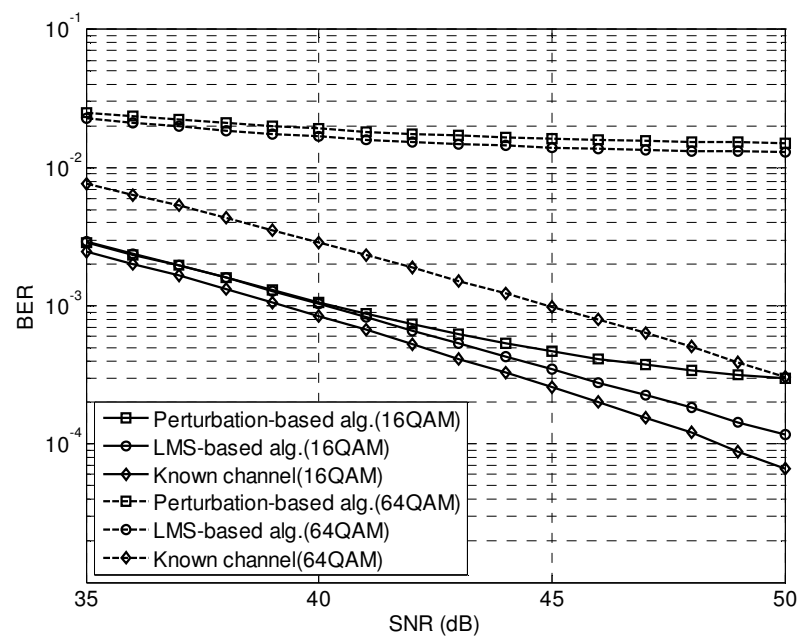

Figure 5. BER versus SNR for $16 Q A M$ and $64 Q A M$ modulations with $M=N=4$.

\section{CONCLUSION}

In this paper the performances of two adaptive SVD estimation algorithms for MIMO-OFDM systems have been evaluated and also compared. Simulation results show that in the perturbation-based algorithm, singular matrices deviate from unitary property which leads to less performance at high SNRs, in comparison with LMS-based algorithm. Meanwhile, although both algorithms have the same convergence rate, due to making some approximations, the computational complexity of perturbation-based algorithm is lower than that of the LMS - based algorithm. Also, these approximations cause the perturbation-based algorithm to have less SINR in comparison with LMS-based algorithm at high SNRs. The difference in BER performances of two algorithms is not significant in BPSK and QPSK modulation schemes, but the perturbation-based algorithm shows higher BER (less performance) at high SNRs for 16QAM and 64QAM modulation schemes.

\section{ACKNOWLEDGMENT}

This work was supported in part by Iran Telecom Research Center (ITRC).

\section{REFERENCES}

[1] Y. (G.) Li, "Simplified channel estimation for OFDM systems with multiple transmit antennas," IEEE Trans. Wireless Commun., vol. 1, Jan. 2002.

[2] H. Sempath, P. Stoica and A. Paularj, "Generalized linear precoder and coder design for MIMO channels using the weighed MMSE criterion," IEEE Trans. Commun., vol. 49, no. 12, pp. 2198-2206, Dec. 2001.

[3] G. Q. Sun, D. C. Cox, H. C. Huanq and A. Lozano, "Estimation of continuous flat fading MIMO channels," IEEE Trans. Wireless Commun., vol. 1, pp. 449-553, Oct. 2002.

[4] T. J. Willink, "Efficinet adaptive SVD algorithm for MIMO applications", IEEE Trans. on Signal Processing, vol. 56, no. 2, pp. 615622, Feb. 2008.

[5] H. Zamiri-Jafarian and G. Gulak, "Adaptive channel SVD estimation for MIMO-OFDM systems," in Proc. VTC 2005, vol. 1, pp. 552-556, June 2005 .

[6] B. Farhang-Boroujeny, Adaptive Filters: Theory and Applications. New York: John Wiley, 1998. 